\title{
AVALIAÇÃO DE CRITÉRIOS PARA CALAGEM DE ARROZ INUNDADO EM CASA DE VEGETAÇÃO(1)
}

\author{
M. BORGES J ÚNIOR (2), J . W. V. MELLO(3), \\ A. C. RIBEIRO ${ }^{(3)} \&$ P. C. SOARES ${ }^{(4)}$
}

\begin{abstract}
RESUMO
O presente trabalho foi desenvolvido, no período de maio a setembro de 1995, com o objetivo de avaliar critérios para a recomendação de calcário para o arroz em solos inundados de Minas Gerais. Foram avaliados os efeitos de doses crescentes de carbonatos de cálcio e de magnésio sobre a produção de matéria seca de plantas de arroz em casa de vegetação. 0 experimento constou de um fatorial completo com dez solos de várzeas e cinco níveis de calagem. Verificouse que a acidez potencial (H + Al) a pH 7,0, a saturação por bases (V), o teor de carbono orgânico (C) e o teor de cálcio mais magnésio $(\mathrm{Ca}+\mathrm{Mg})$ foram as características dos solos que mais influenciaram as doses recomendáveis de calcário. Por outro lado, os teores de Fe e de Mn redutíveis do solo não tiveram influência significativa na recomendação de calagem. 0 método que visa elevar V a $\mathbf{4 0 \%}$ foi mais eficiente na definição das doses recomendáveis para obtenção da matéria seca equivalente a $\mathbf{9 0 \%}$ da máxima eficiência física.
\end{abstract}

Termos de indexação: solos alagados, recomendação de calcário, toxicidade de Fe.

\section{SUMMARY: LIMING CRITERIA EVALUATION FOR WATERLOGGED RICE IN GREENHOUSE}

This research was conducted from May to September, 1995, in order to eval uateli ming criteria for rice pl ants on flooded soi ls of M inas Gerais State, Brazi l. The effects of increasing $\mathrm{CaCO}_{3}+\mathrm{MgCO}_{3}$ dosis on dry matter production were evaluated in a greenhouse pot trial. The experimental matrix was a complete factorial with ten lowland soils and five levels of liming. The soil characteristics which significantly affected the lime levels recommended

\footnotetext{
(1) Parte da Tese de Mestrado do primeiro autor, apresentada ao Departamento de Sol os, Universidade Federal de Viçosa. Recebido para publicação em junho de 1997 e aprovado em abril de 1998.

(2) Químico Industrial, Mestre em Solos e Nutrição de Plantas, Universidade Federal de Viçosa. CEP 36571-000 Viçosa (MG).

(3) Professor do Departamento de Solos, Universidade Federal Viçosa.CEP 36571-000 Viçosa (MG).

(4) Pesquisador da EPAMIG. CEP 36570-000 Viçosa (MG).
} 
were: titrableacidity at $\mathrm{pH} 7,0(\mathrm{H}+\mathrm{Al})$, base saturation $(\mathrm{V})$, content of organic carbon $(\mathrm{C})$ and contents of calcium plus magnesium $(\mathrm{Ca}+\mathrm{Mg})$. On the other hand, the contents of reducibleFeand $\mathrm{Mn}$ did not significantly influencetheliming recommendation. Theliming method for increasing base saturation to $40 \%$ was more effective in determining the recommended doses for attaining a dry matter production of $90 \%$ of maxi mum yields.

Index terms: flooded soils, liming recommendation, iron toxicity.

\section{INTRODUÇÃO}

Quatrosistemas decultivo de arroz são utilizados no Brasil, de acordo com as condições econômicas e topográficas de cada região e com o suprimento de água: arroz de sequeiro, que ocupa a maior área; arroz irrigado por inundação controlada, que apresenta maior rendimento; arroz irrigado por inundação natural, earroz de baixada úmida. Mesmo ocupando, aproximadamente, apenas $20 \%$ das áreas cultivadas, os sistemas de cultivo de arroz por inundação respondem por cerca de $40 \%$ da produção (Fageria et al., 1984). Entretanto, o cultivo de arroz por inundação traz como conseqüências várias alterações físico-químicas que influem na dinâmica ena disponi bilidade das espécies quími cas presentes no solo.

Dentreas principais limitações ao cultivo de arroz em sol os inundados, destaca-se a toxicidade por ferro (Tanaka et al., 1966; Breemen \& Moormann, 1977, citados por Fageria et al., 1981). Os distúrbios fisiológicos atribuídos a essa toxicidade têm provocado grandes perdas na produção de arroz, em virtude de estarem associados com a diminuição dos índices de produtividade (Fageria et al., 1984). Segundo Ottow et al. (1982) e Vahl (1991), a toxicidade de ferro é mais uma questão de balanço nutricional do que uma conseqüência de altas concentrações de Fe redutível no solo.

Em solos inundados, verifica-se a redução de óxidos, hidróxidos e oxidróxidos de ferro, de modo que as concentrações de íons $\mathrm{Fe}^{2+}$ em solução podem atingir níveis tóxicos. Segundo Howeler (1973), os teores de Fe em solução podem ser diminuídos por vários tratamentos de manejo de água e pela adição de grandes quantidades de cal cário. Outros autores também consi deram a calagem como prática viável para minimizar os problemas de toxicidade de ferro e de manganês (Barbosa Filho, 1983; Freire et al., 1985; Ramos et al., 1985; Barbosa Filho, 1988), bem como para suprir as necessidades de $\mathrm{Ca}$ e de $\mathrm{Mg}$ para arroz inundado.

Diferentes métodos têm sido utilizados na determinação da necessidade de cal cário: saturação por bases (Raij et al., 1981), acidez potencial (Malavolta, 1959), alumínio trocável (Nye et al., 1961), alumínio trocável mais cálcio e magnésio (Comissão de Fertilidade do Solo do Estado de Minas
Gerais, 1978), matéria orgânica elevando o pH para 6,5 (Keeney \& Corey, 1963), matéria orgânica el evando o pH para 6,0 (Defelipo et al., 1972), SMP (Shoemaker et al., 1961) e incubação com doses crescentes de carbonato de cál cio (Souza et al., 1980), que é utilizado como método-padrão. $O$ método da incubação é empregado apenas em pesquisas e na calibração dos demais métodos, por ser muito demorado para a utilização em laboratórios de rotina. Esses métodos variam em seus princípios analíticos e, dessa forma, conduzem a diferentes quantidades de cal cário recomendadas para um mesmo solo.

São muito controvertidos os resultados da resposta de arroz à calagem. Leite et al. (1970) não observaram resposta de plantas de arroz, sob sistema irrigado, à apl icação de cal cário dolomítico, nas doses de 3 a $5 \mathrm{t} \mathrm{ha}^{-1} \mathrm{em}$ solos ácidos do Vale do Paraíba. Entretanto, deve-se salientar que esses solos apresentavam teores de $\mathrm{Ca}+\mathrm{Mg}$ na faixa de 2,5 a $7,0 \mathrm{cmol} \mathrm{dm}^{-3}$. Também Schmidt \& Gargantini (1970) nấo observaram aumento na produção de matéria seca e de grãos de arroz, cultivado sob inundação, com aplicação de 6 t ha-1 de cal cário. Por outro lado, Machado et al . (1984) e Machado (1985) obtiveram aumento na produção de arroz pela adição de calcário. Esses autores, porém, não souberam definir se a resposta foi devida à correção da acidez do solo ou à deficiência de Ca e Mg. Também, Kaminski et al. (1990) obtiveram respostas significativas à adição de cal cário dol omítico em sol o de várzea, com acréscimo na produção de grãos no primeiro e no segundo ano. Esse solo, porém, apresentava teores de $5 \mathrm{cmol}_{\mathrm{C}} \mathrm{dm}^{-3}$ deAl e menos de $3 \mathrm{cmol}_{\mathrm{C}} \mathrm{dm}^{-3} \mathrm{de} \mathrm{Ca}+\mathrm{Mg}$.

Em vista do exposto, o trabalho foi desenvolvido com os objetivos de avaliar não só os efeitos de doses crescentes de cal cário sobre a produção de matéria seca de plantas de arroz, mas também os critérios para a recomendação de calagem em solos inundados.

\section{MATERIAL E MÉTODOS}

Este trabalho constou de um experimento com plantas de arroz (Oryza sativa, L.) em dez solos incubados com doses crescentes de calcário, 
submetidos à inundação em casa de vegetação. $\mathrm{O}$ experimento foi realizado, no período de maio a setembro de 1995, nas dependências do Departamento de Sol os da U niversidade Federal de Viçosa.

Foram col etadas amostras superficiais $(0-20 \mathrm{~cm})$ de dez solos de várzeas do Estado de Minas Gerais. A pós serem secas ao ar, as amostras foram destorroadas, tamisadas em peneiras de $2,0 \mathrm{~mm}$ de abertura de malha e caracterizadas física e quimicamente (Quadros 1 e 2): anál ise textural (Método da pipeta; EMBRAPA, 1979); pH; fósforo, potássio e sódio (extrator Mehlich-1); cálcio, magnésio e alumínio (extrator $\mathrm{KCl} 1 \mathrm{~mol} \mathrm{L-1}$; EMBRAPA, 1979); acidez potencial (extrator $\mathrm{Ca}(\mathrm{OAc})_{2}$ 0,5 mol L-1 pH 7,0), materia orgânica (Walkley-Black); ataque sulfúrico (Vettori, 1969) e teores de Fe e Mn pelos extratores de Mehlich-1 e DTPA-trietanolamina (Lindsay e Norvel, 1978), respectivamente. Posteriormente, as amostras foram subdi vididas em porções de 2,5 $\mathrm{dm}^{3}$ eacondicionadas em sacos de polietileno para receberem os tratamentos pertinentes.
Amostras de 2,5 dm $\mathrm{dm}^{3}$ de sol o foram incubadas, em sacos plásticos, com doses crescentes de corretivo $\left(\mathrm{CaCO}_{3}+\mathrm{MgCO}_{3}\right.$, relação molar 4:1), por um período de 60 dias. Nesse período, a umidade foi mantida próxima a $80 \%$ da capacidade de campo dos solos, determinada pelo método da coluna transparente (Fernandes, 1967). As doses de corretivo corresponderam a $0 ; 0,4 ; 0,8 ; 1,2$ e 1,6 vezes a necessidade de calagem (NC) dos sol os, determinada a partir da acidez potencial a pH 7,0 $(\mathrm{H}+\mathrm{Al})$, sendo $\mathrm{NC}$ (t ha-1) $=\mathrm{H}+\mathrm{Al}\left(\mathrm{cmol}_{\mathrm{c}} \mathrm{dm}^{-3}\right)$. $\mathrm{O}$ delineamento experimental adotado foi o de blocos casualizados com três repetições, utilizando-se um esquema fatorial completo de cinco níveis de calagem e dez solos.

Amostras de $2 \mathrm{dm}^{3}$ de solo incubado foram transferidas para vasos de $3 \mathrm{~L}$, onde foram plantadas cinco mudas de arroz (cultivar PR 477), prégerminadas em leito de areia. Após o 3ㅇ dia do transplante, os vasos foram inundados, mantendose, a partir daí, uma lâmina d'água de $3 \mathrm{~cm}$ sobre a superfície do solo.

Quadro 1. Procedência, classificação e análise textural ${ }^{(1)}$ dos solos de Minas Gerais utilizados

\begin{tabular}{|c|c|c|c|c|c|c|}
\hline \multirow{2}{*}{ Solo } & \multirow{2}{*}{ Procedência } & \multirow{2}{*}{ Classificação } & \multicolumn{2}{|c|}{ Areia } & \multirow{2}{*}{ Silte } & \multirow{2}{*}{ Argila } \\
\hline & & & Fina & $\overline{\text { Grossa }}$ & & \\
\hline & & & \multicolumn{4}{|c|}{$\mathrm{g} \mathrm{kg}^{-1}$} \\
\hline O-La & Lambari & Orgânico & 70 & 90 & 310 & 530 \\
\hline $\mathrm{GH}-\mathrm{L} a$ & Lambari & Glei Húmico & 140 & 140 & 230 & 490 \\
\hline GPH-La & Lambari & Glei Pouco Húmico & 70 & 200 & 250 & 480 \\
\hline $\mathrm{GPH}-\mathrm{V}$ & Viçosa & Glei Pouco Húmico & 70 & 280 & 230 & 420 \\
\hline$A L-L 2$ & Leopoldina & Aluvial & 380 & 80 & 120 & 420 \\
\hline$A L-C$ & Cambuquira & Aluvial & 180 & 300 & 140 & 380 \\
\hline$A L-V$ & Viçosa & Aluvial & 170 & 360 & 220 & 250 \\
\hline PV-V & Viçosa & Podzólico V. Amarelo & 30 & 60 & 180 & 730 \\
\hline GPH-M & Muriaé & Glei Pouco Húmico & 70 & 110 & 210 & 610 \\
\hline$A L-L 1$ & Leopoldina & Aluvial & 480 & 90 & 110 & 320 \\
\hline
\end{tabular}

(1) Método da pipeta (EMBRAPA, 1979).

Quadro 2. Algumas características químicas dos solos de Minas Gerais utilizados

\begin{tabular}{|c|c|c|c|c|c|c|c|c|c|c|c|c|c|c|c|c|}
\hline \multirow{2}{*}{ Solo } & \multirow{2}{*}{$\begin{array}{c}\mathbf{p H} \\
\mathrm{H}_{2} \mathrm{O}\end{array}$} & \multirow{2}{*}{$\mathbf{P}$} & \multirow[b]{2}{*}{$\mathbf{K}$} & \multirow[b]{2}{*}{$\mathrm{Na}$} & \multirow[b]{2}{*}{$\mathbf{C a}$} & \multirow[b]{2}{*}{ Mg } & \multirow[b]{2}{*}{$\mathbf{A l}$} & \multirow[b]{2}{*}{$\mathbf{H}+\mathbf{A l}$} & \multirow{2}{*}{$\mathbf{T}^{(\mathbf{1})}$} & \multirow{2}{*}{ M.O. } & \multicolumn{4}{|c|}{ Ataque Sulfúrico } & \multirow{2}{*}{ Fe-Mehlich-1 } & \multirow{2}{*}{ Mn-DTPA } \\
\hline & & & & & & & & & & & $\mathrm{Fe}_{2} \mathrm{O}_{3}$ & $\mathrm{Al}_{2} \mathbf{O}_{3}$ & $\mathrm{TiO}_{2}$ & $\mathrm{SiO}_{2}$ & & \\
\hline & & $\mathrm{mg} \mathrm{kg}^{-1}$ & & & 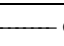 & $\mathrm{mol}_{\mathrm{C}} \mathrm{C}$ & $m^{-3}$ & & - & 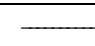 & 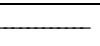 & dag $\mathrm{kg}^{-1}$ & & & $\ldots \mathrm{mg} \mathrm{d}$ & $n^{-3}$ \\
\hline O-La & 4,5 & 3,77 & 0,11 & 0,18 & 0,7 & 0,2 & 2,1 & 20,8 & 22,0 & 19,96 & 1,3 & 19,8 & 0,4 & 16,7 & 22 & 15 \\
\hline GH-La & 4,3 & 5,48 & 0,28 & 0,16 & 0,4 & 0,3 & 2,5 & 15,5 & 16,6 & 11,38 & 2,9 & 18,8 & 0,5 & 16,3 & 45 & 27 \\
\hline GPH-LE & 4,3 & 6,14 & 0,10 & 0,16 & 0,1 & 0,1 & 2,4 & 11,1 & 11,6 & 6,90 & 3,7 & 16,7 & 0,5 & 17,8 & 229 & 36 \\
\hline GPH-V & 5,5 & 2,79 & 0,07 & 0,11 & 1,2 & 0,3 & 0,5 & 7,4 & 9,1 & 8,79 & 11,1 & 17,2 & 0,9 & 15,6 & 1.968 & 501 \\
\hline$A L-L 2$ & 4,8 & 9,30 & 0,16 & 0,28 & 0,7 & 0,4 & 0,2 & 5,3 & 6,8 & 2,93 & 3,5 & 16,8 & 0,5 & 15,4 & 1.037 & 41 \\
\hline$A L-C$ & 5,9 & 7,55 & 0,72 & 1,13 & 0,7 & 0,3 & 0,0 & 4,3 & 7,2 & 3,76 & 3,3 & 9,0 & 0,3 & 6,8 & 1.218 & 60 \\
\hline$A L-V$ & 5,7 & 1,25 & 0,18 & 0,31 & 2,5 & 1,0 & 0,0 & 3,5 & 7,4 & 3,48 & 7,6 & 13,7 & 0,9 & 13,1 & 1.727 & 320 \\
\hline$P V-V$ & 5,4 & 2,84 & 0,12 & 0,19 & 2,7 & 1,0 & 0,1 & 5,5 & 9,4 & 4,07 & 11,6 & 28,4 & 0,9 & 24,9 & 92 & 329 \\
\hline GPH-M & 6,0 & 2,48 & 0,10 & 0,15 & 3,8 & 1,1 & 0,0 & 4,5 & 9,6 & 5,78 & 8,5 & 25,2 & 0,8 & 23,1 & 1.605 & 318 \\
\hline AL-L 1 & 5,5 & 3,77 & 0,11 & 0,19 & 1,8 & 1,0 & 0,1 & 2,0 & 5,0 & 2,28 & 2,6 & 14,6 & 0,4 & 12,1 & 702 & 49 \\
\hline
\end{tabular}

(1) $\mathrm{T}=\mathrm{Ca}+\mathrm{Mg}+\mathrm{K}+\mathrm{Na}+(\mathrm{H}+\mathrm{Al})$. 
Foram real izadas adubações com $\mathrm{N}\left(200 \mathrm{mg} \mathrm{dm}^{-3}\right)$, P (300 mg dm-3$), ~ K ~\left(150 ~ ~ m g ~ d m^{-3}\right), S\left(40 \mathrm{mg} \mathrm{dm}^{-3}\right)$, Zn (6 mg dm-3), Cu (3 mg dm-3), B (0,81 mg dm-3) eMo $\left(0,15 \mathrm{mg} \mathrm{dm}^{-3}\right)$. O P e o $\mathrm{K}$ foram adicionados ao plantio, e o $\mathrm{N}$ foi parcelado (Mello, 1991), adicionando-se $50 \mathrm{mg} \mathrm{dm}^{-3}$ no plantio e $50 \mathrm{mg} \mathrm{dm}^{-3}$ a cada dez dias. J á os mi cronutrientes foram parcelados, colocando-se a metade das doses no plantio, e o restante após 20 dias. O corte das plantas foi feito na época de máximo perfilhamento, mas antes da diferenciação do primórdio floral, 55 dias após o transplante das mudas. O material vegetal foi seco em estufa com ventilação forçada a $70^{\circ} \mathrm{C}$, até peso constante e pesado.

As análises de variância foram realizadas, separadamente, para cada solo, uma vez que não houve homogeneidade das variâncias. Foram realizados estudos de regressão, para a produção de matéria seca em função das doses de calcário, tendo sido testados os model os linear, raiz quadrada, quadrático e cúbico. Os model os foram sel ecionados, obedecendo aos crité rios de comparação dos coeficientes de determinação ajustados e de significância dos coeficientes deregressão pelo teste " $\mathrm{t}$ " de Student, considerando-se os níveis de significância de 10, 5 e 1\% de probabilidade.

A partir das equações de regressão para a matéria seca em função das doses de cal cário, foram calculadas a produção máxima de matéria seca estimada dentro dointervalo experimental eas doses decal cário necessárias para se obter produção de matéria seca equivalente a 90, 95 e 100\% da máxima eficiência física (MEF). Foram obtidos, ainda, modelos de regressão para a previsão das doses recomendadas de cal cário em função de al gumas características químicas dos solos.

As doses recomendadas para 90, 95 e 100\% da MEF foram comparadas com a necessidade de calcário cal culada pelos métodos que utilizam como critério o teor de matéria orgânica para elevar o pH a 6,5 (Keeney \& Corey, 1963), o pH a 6,0 (Defelipo et al., 1972), a saturação por bases para el evar V a 70\% (Raij et al., 1981) e para elevar V a 40\% (Comissão de Fertilidade do Solo doE stado de Minas Gerais, 1978), o teor de alumínio trocável (Nye et al., 1961), a acidez potencial (Malavolta, 1959), e o método baseado nos teores deAl trocável mais cálcioemagnésio (Comissão de Fertilidade do Solo do Estado de Minas Gerais, 1978). Para comparar as necessidades de calcário, ajustaram-se equações de regressão, com as doses recomendadas como variável dependente $(\hat{y})$ das necessi dades de cal cário pel os vários métodos, pois se trata de predizer o recomendável em função dos métodos utilizados. Embora não exista uma relação de dependência entre essas variáveis, tal artifício estatístico foi utilizado para indicar qual o melhor método de recomendação de cal cário. Dessa forma, por meio do coeficiente de correlação $(r)$ e do coeficiente angular da equaçãoderegressão, pode-seter uma idéia se as doses recomendadas foram adequadas, subestimadas ou superestimadas em relação às necessidades de cal cário calculadas pelos diferentes métodos.

\section{RESULTADOS E DISCUSSÃO}

De modo geral, as plantas apresentaram sintomas visuais detoxicidade de Fena dosezero de cal cário. O excesso de ferro solúvel retardou o crescimento das plantas. As fol has mais vel has apresentaram bronzeamento, expandindo-se da extremidade superior para a base, advindo depois o secamento ea morte dessas fol has. Sintomas semel hantes foram descritos por Tanaka et al. (1966). Esses sintomas visuais foram reduzidos e até eliminados, à medida que as doses de cal cário aumentaram. A calagem tem sido considerada, por vários autores, uma prática eficiente no controle da toxicidade de Fe para o arroz em sol os inundados (Ramos et al. 1985; Barbosa Filho, 1988). Entretanto, sabe-se que a aplicação de cal cário em excesso pode promover a deficiência de vários micronutrientes. A esse respeito, verificaram-se sintomas de clorose internerval, com a morte de algumas plantas, para as duas doses de calcário mais elevadas nos solos AL-L $1, A L-V$ e AL-C, durante o período inicial de crescimento. A dorose desapareceu gradativamenteapós a segunda semana deinundação dos solos, evidenciando a deficiência temporária de ferro. Em cultivos em casa de vegetação, Mello (1991) verificou que a calagem promoveu aumento no $\mathrm{pH}$ dos solos e decréscimos nas concentrações de $\mathrm{Fe}^{2+} \mathrm{e}$ $\mathrm{Mn}^{2+}$ em sol ução. Seus resultados demonstraram que pequenas quantidades de calcário foram suficientes para promover a diminuição dos teores de Fe em solução. Também F reire et al. (1985) observaram que a calagem foi a prática mais eficiente no controle da toxicidade do ferro, em relação à adubação orgânica e ao manejo da água.

A produção de matéria seca da parte aérea das plantas variou deacordo com os sol os estudados ecom as doses de cal cário aplicadas (Quadros 3 e 4). Nos solos AL-L1, AL-V, AL-C, GH-La, PV-V e GPH-V, a produção de matéria seca obtida na dose de cal cário mais al ta foi inferior àquela obtida na dosezero. Isso pode ser atribuído às limitações, causadas pela calagem, na disponibilidade de micronutrientes, principalmente de ferro. Verificou-se, ainda, a diminuição dos sintomas visuais de toxicidade de $\mathrm{Fe}$, conjuntamente com o aumento do peso da matéria seca.

As mai ores doses recomendadas de cal cári o foram obtidas para os sol os O-La, GH-La, GPH-La eGPH-V, queapresentaram mai ores teores decarbono orgânico e menores porcentagens de saturação por bases (Quadro 5). Os coeficientes de determinação das equações de regressão para as doses recomendadas de calcário, considerando algumas características quími cas dos solos (Quadro 6), foram maiores para as equações nas quais seutilizaram doses recomendadas para $100 \%$ da MEF, como variável dependente. Adicionalmente, verificou-se que o uso dos teores de $\mathrm{Fe}$, obtido pelo extrator Mehlich-1, e de $\mathrm{Mn}$, pelo extrator DTPA-trietanolamina, não apresentou efeito significativo nos model os preditivos. Isso indica quea 
Quadro 3. Produção de matéria seca da parte aérea de plantas de arroz (g vaso-1) para os diferentes níveis de calagem

\begin{tabular}{|c|c|c|c|c|c|}
\hline \multirow{2}{*}{ Solo } & \multicolumn{5}{|c|}{ Níveis de Calcário(1) } \\
\hline & $\mathbf{0}$ & 0,4 & 0,8 & 1,2 & 1,6 \\
\hline & & & $\mathrm{g}$ vaso & & \\
\hline $\mathrm{O}-\mathrm{La}$ & 15,9 & 20,3 & 25,1 & 20,4 & 17,6 \\
\hline $\mathrm{GH}-\mathrm{La}$ & 22,0 & 23,0 & 27,3 & 23,6 & 20,6 \\
\hline GPH-La & 18,0 & 19,9 & 22,0 & 20,7 & 18,4 \\
\hline GPH -V & 21,0 & 24,8 & 27,7 & 24,1 & 19,4 \\
\hline$A L-L 2$ & 21,2 & 24,1 & 25,9 & 22,8 & 22,2 \\
\hline$A L-C$ & 19,0 & 24,1 & 23,3 & 9,6 & 4,2 \\
\hline$A L-V$ & 21,2 & 27,6 & 24,2 & 17,2 & 14,2 \\
\hline$P V-V$ & 26,0 & 27,4 & 29,4 & 27,9 & 23,5 \\
\hline GPH-M & 22,6 & 32,6 & 29,0 & 26,5 & 22,7 \\
\hline$A L-L 1$ & 14,3 & 23,3 & 18,0 & 15,2 & 7,0 \\
\hline
\end{tabular}

${ }^{(1)}$ Níveis de calagem equivalentes a $0 ; 0,4 ; 0,8 ; 1,2$ e 1,6 vezes a acidez potencial $(\mathrm{H}+\mathrm{Al})$ de cada solo.
Quadro 4. Equações de regressão para a produção de matéria seca (MS, g vaso-1) em função das doses de calcário (Cal, $\mathrm{t}$ ha-1)

\begin{tabular}{|c|c|c|}
\hline Solo & E quação de Regressão & $\mathbf{R}^{2}$ \\
\hline O-La & $\mathrm{MS}=15,71+0,867 * * \mathrm{Cal}-0,0248^{* *} \mathrm{Cal}^{2}$ & 0,8653 \\
\hline GH-La & $\mathrm{MS}=21,44+0,707^{\circ} \mathrm{Cal}-0,0299 * \mathrm{Cal}^{2}$ & 0,7478 \\
\hline GPH-Lì & $\mathrm{MS}=17,91+0,742 * \mathrm{Cal}-0,0405 * \mathrm{Cal}^{2}$ & 0,9153 \\
\hline GPH-V & $\mathrm{MS}=20,82+2,122 * \mathrm{Cal}-0,1896 * * \mathrm{Cal}^{2}$ & 0,9588 \\
\hline$A L-L 2$ & $\mathrm{MS}=21,37+1,649 * \mathrm{Cal}-0,1916 * \mathrm{Cal}^{2}$ & 0,7680 \\
\hline$A L-C$ & MS $=18,54+9,019 * * \mathrm{Cal}-3,2339 * * \mathrm{Cal}^{2}+0,234 * * \mathrm{Cal}^{3}$ & 0,9626 \\
\hline$A L-V$ & MS $=21,17+10,023 * \mathrm{Cal}-4,4375 * \mathrm{Cal}^{2}+0,433 * \mathrm{Cal}^{3}$ & 0,9998 \\
\hline$P V-V$ & $\mathrm{MS}=25,54+1,772^{\circ} \mathrm{Cal}-0,2266^{\circ} \mathrm{Cal}^{2}$ & 0,9201 \\
\hline GPH-M & $\mathrm{MS}=22,72+13,621^{* *} \mathrm{Cal}^{1 / 2}-5,1588^{* *} \mathrm{Cal}$ & 0,9721 \\
\hline$A L-L 1$ & MS $=14,29+22,922 * * \mathrm{Cal}^{1 / 2}-15,0542 * * \mathrm{Cal}$ & 0,9722 \\
\hline
\end{tabular}

**, $*{ }^{\circ}$ Significativo a 1,5 e $10 \%$ de probabilidade, respectivamente.

Quadro 5. Estimativas das produções de máxima eficiência física (MEF - 90, 95 e 100\%), doses recomendadas de calcário (DR) e algumas características químicas dos solos

\begin{tabular}{|c|c|c|c|c|c|c|c|c|c|}
\hline Solo & $90 \%$ ME F & $D R^{(1)}$ & 95\% ME F & $\mathbf{D R}{ }^{(2)}$ & $100 \%$ ME F & $D R^{(3)}$ & $C^{(4)}$ & $\mathbf{V}^{(5)}$ & $\mathrm{Ca}+\mathrm{Mg}^{(6)}$ \\
\hline & g vaso-1 & $\mathrm{t} \mathrm{ha}^{-1}$ & g vaso-1 & $\mathrm{t} \mathrm{ha}^{-1}$ & g vaso-1 & t ha-1 $^{-1}$ & dag kg-1 & \multicolumn{2}{|c|}{$\%$} \\
\hline $\begin{array}{l}\text { O-La } \\
\text { GH-La } \\
\text { GPH-La } \\
\text { GPH-V } \\
\text { AL-L2 } \\
\text { AL-C } \\
\text { AL-V } \\
\text { PV-V } \\
\text { GPH-M } \\
\text { AL-L1 }\end{array}$ & $\begin{array}{l}20,97 \\
23,05 \\
19,18 \\
24,09 \\
22,45 \\
23,11 \\
24,92 \\
26,11 \\
28,54 \\
20,71\end{array}$ & $\begin{array}{l}7,80 \\
2,56 \\
1,91 \\
1,84 \\
0,70 \\
0,65 \\
0,47 \\
0,33 \\
0,29 \\
0,14\end{array}$ & $\begin{array}{l}22,14 \\
24,33 \\
20,24 \\
25,42 \\
23,67 \\
24,40 \\
26,31 \\
27,56 \\
30,13 \\
21,86\end{array}$ & $\begin{array}{r}10,64 \\
5,27 \\
4,03 \\
2,94 \\
1,75 \\
0,95 \\
0,74 \\
1,38 \\
0,59 \\
0,24\end{array}$ & $\begin{array}{l}23,30 \\
25,61 \\
21,31 \\
26,76 \\
24,91 \\
25,68 \\
27,69 \\
29,01 \\
31,71 \\
23,01\end{array}$ & $\begin{array}{r}17,51 \\
11,81 \\
9,16 \\
5,60 \\
4,30 \\
1,71 \\
1,43 \\
3,91 \\
1,74 \\
0,58\end{array}$ & $\begin{array}{r}11,58 \\
6,60 \\
4,00 \\
5,10 \\
1,70 \\
2,18 \\
2,02 \\
2,36 \\
3,35 \\
1,32\end{array}$ & $\begin{array}{r}5,28 \\
6,73 \\
4,66 \\
18,08 \\
22,01 \\
40,08 \\
53,11 \\
42,14 \\
53,55 \\
71,39\end{array}$ & $\begin{array}{c}3,96 \\
4,08 \\
2,41 \\
16,1 \\
15,51 \\
14,25 \\
46,49 \\
38,85 \\
50,94 \\
54,31\end{array}$ \\
\hline
\end{tabular}

(1) Dose recomendada para 90\% MEF ; ${ }^{(2)}$ Dose recomendada para 95\% MEF; ${ }^{(3)}$ Dose recomendada para $100 \%$ MEF; ${ }^{(4)}$ Carbono orgânico (C) (Walkley -Black); ${ }^{(5)}$ Saturação por bases $(\mathrm{V})=(\mathrm{Ca}+\mathrm{Mg}+\mathrm{K}+\mathrm{Na}) 100 \mathrm{~T}^{-1}$; ${ }^{(6)}$ Saturação por cálcio mais magnésio = $(\mathrm{Ca}+\mathrm{Mg}) 100 \mathrm{~T}^{-1}$.

resposta das plantas à cal agem está mais relacionada com a diminuição da acidez potencial e com o fornecimento de $\mathrm{Ca}$ e $\mathrm{Mg}$, para as plantas do que com a diminuição dos teores de Fe e Mn na solução dos solos, assumindo-se que os teores de Fe e Mn redutíveis foram convenientemente estimados pelos extratores Mehlich-1 e DTPA-trietanolamina, respectivamente. Efetivamente, verificou-se que, mesmo nos solos O-La, GH-La, PVV e GPH-La, com baixos teores de Fe e $\mathrm{Mn}$ redutíveis (Quadro 2), a calagem promoveu aumentos significativos na produção de matéria seca (Quadros 3 e 4). Esses resultados revelam quea toxicidade de ferroé mais uma questão de balanço nutricional do que uma conseqüência dealtas concentrações de Feredutível no solo, conforme admitem Ottow et al. (1982), Barbosa Filho et al. (1983) e Vahl (1991).

Comparando as doses recomendadas de cal cário, obtidas no presente trabalho, com as necessidades de calcário, calculadas para os métodos citados anteriormente, verificou-se que os métodos baseados no teor de matéria orgânica para elevar o pH a 6,0 e 6,5 superestimaram as doses de calcário recomendadas. Isso é demonstrado pelos baixos valores dos coeficientes angulares das equações de regressão (Figuras 1 e2), embora a correlação tenha sido alta $(r>0,94)$. J á os métodos baseados no Al trocável enoAl mais Ca eMg trocável subestimaram as doses recomendáveis para a obtenção de $100 \%$ da ME F, sendo os coeficientes angulares das equações 
Quadro 6. Equações de regressão das doses de calcário (Cal, t ha-1) em função de algumas características químicas dos solos para a obtenção de 90, 95 e 100\% da máxima eficiência física (MEF)

\begin{tabular}{|c|c|}
\hline $\mathbf{E}$ & $\mathbf{R}^{2}$ \\
\hline $\begin{array}{l}(\mathrm{C})-0,9364^{*}(\mathrm{~V}) \\
(\mathrm{C}) \\
(\mathrm{Fe}) \\
(\mathrm{Mn}) \\
(\mathrm{C})-0,4081^{*}(\mathrm{CaMg}) \\
(\mathrm{C})-0,3015^{*}(\mathrm{PCaMg}) \\
(\mathrm{C})-0,0276^{\circ}(\mathrm{V}) \\
(\mathrm{Fe}) \\
(\mathrm{Mn}) \\
{ }^{*}(\mathrm{H}+\mathrm{Al})-0,2781^{\circ}(\mathrm{CaMg}) \\
{ }^{k}(\mathrm{H}+\mathrm{Al})-0,0248^{\circ}(\mathrm{V}) \\
{ }^{\circ}(\mathrm{H}+\mathrm{Al})-0,2364^{\circ}(\mathrm{PCaMg}) \\
(\mathrm{Fe}) \\
(\mathrm{Mn})\end{array}$ & $\begin{array}{l}0,1387 \\
0,9889 \\
0,9885 \\
0,9885 \\
0,3971 \\
0,1567\end{array}$ \\
\hline
\end{tabular}

**,*, : Significativo ao nível de 1, 5 e $10 \%$ de probabilidade, respectivamente. ns = Não significativo até o nível de $10 \%$ de probabilidade. ${ }^{(1)}$ Para a obtenção de $90 \%$ da MEF. ${ }^{(2)}$ Para obtenção de $95 \%$ da MEF. ${ }^{(3)}$ Para obtenção de $100 \%$ da MEF. de regressão maiores que 1,7 (Figuras 3 e 4). Esses métodos, também, nãosão bons para estimar as doses recomendadas para 90 e 95\% da MEF, em razão dos baixos coeficientes de correlação $(r<0,90)$ e baixas capacidades preditivas $\left(R^{2}<0,70\right)$.

$\mathrm{O}$ método baseado na acidez potencial $(\mathrm{H}+\mathrm{Al})$ demonstrou ser bom estimador das doses de cal cário recomendadas para obtenção de $100 \%$ da MEF, com coeficienteangular deregressão próximoa 1,0 (Figura 5), apresentando, também, alta correlação $(r=0,99)$. Entretanto, para 90 e $95 \%$ da MEF esse método superestimou as doses recomendadas. O método que considera a saturação por bases (para el evar V a 70\%) superestimou os valores para 90 e $95 \%$ da MEF (coeficientes angulares de 0,46 e 0,68 respectivamente) e subestimou para $100 \%$ (coeficiente angular $=1,2$ ), apresentando, porém, alta correlação (Figura 6). Por outro lado, o método que visa el evar a saturação por bases a $40 \%$ foi mais eficiente para estimar as doses recomendáveis de calcário para obtenção de $95 \%$ da MEF (coeficiente angular $=1,1$ ), apresentando alta correlação (Figura 7).

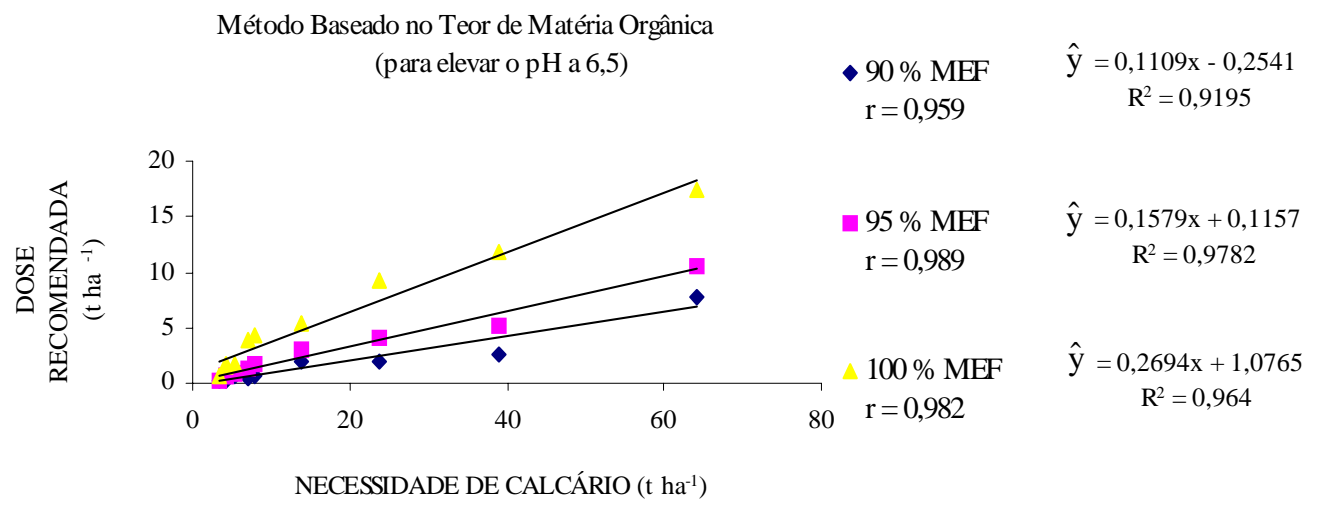

Figura 1. Relação entre as necessidades de calcário, pelo método que considera o teor de matéria orgânica (para elevar o pH a 6,5), e as doses recomendadas de calcário para obtenção de 90,95 e $100 \%$ da máxima eficiência física (MEF).

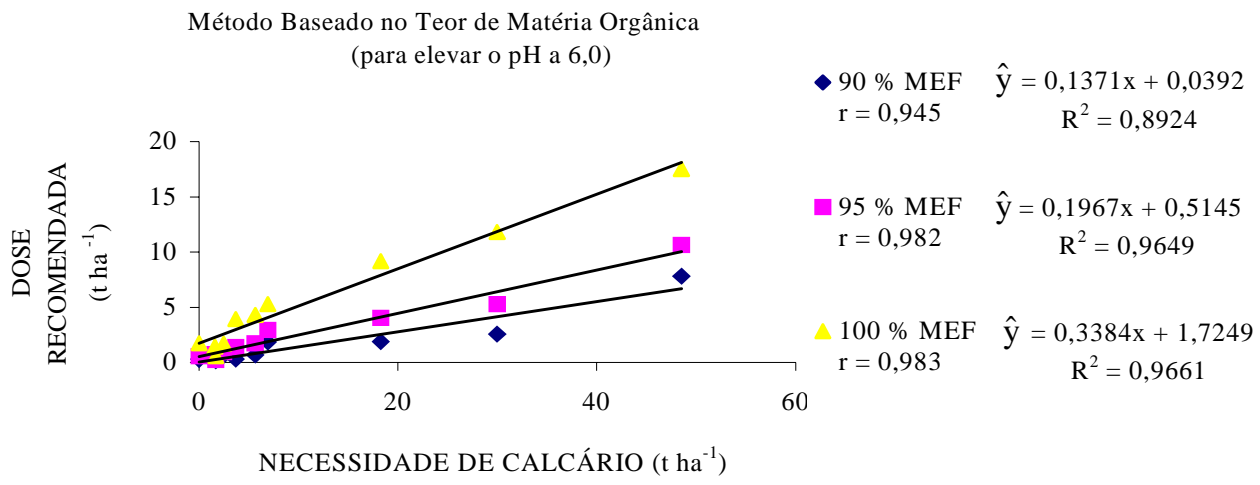

Figura 2. Relação entre as necessidades de calcário, pelo método que considera o teor de matéria orgânica (para elevar o pH a 6,0), e as doses recomendadas de calcário para obtenção de 90, 95 e 100\% da máxima eficiência física (MEF). 
Método Baseado no Teor de Alumínio Trocável

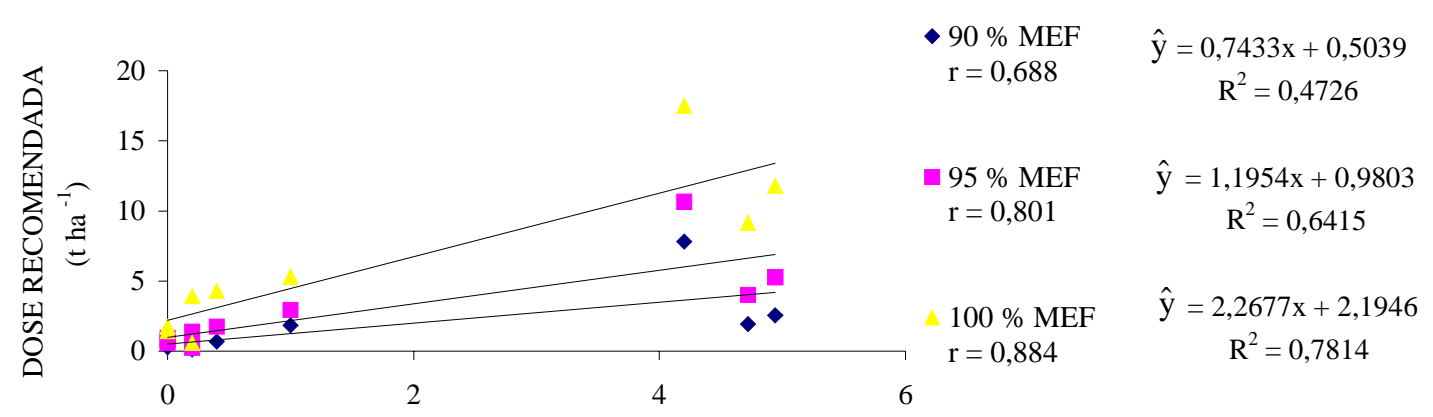

NECESSIDADE DE CALCÁRIO $\left(\mathrm{t} \mathrm{ha}^{-1}\right)$

Figura 3. Relação entre as necessidades de calcário, pelo método baseado no alumínio trocável, e as doses recomendadas de calcário para obtenção de 90, 95 e 100\% da máxi ma eficiência física (ME F).

Método Baseado no Teor de Alumínio Trocável mais Cálcio e Magnésio

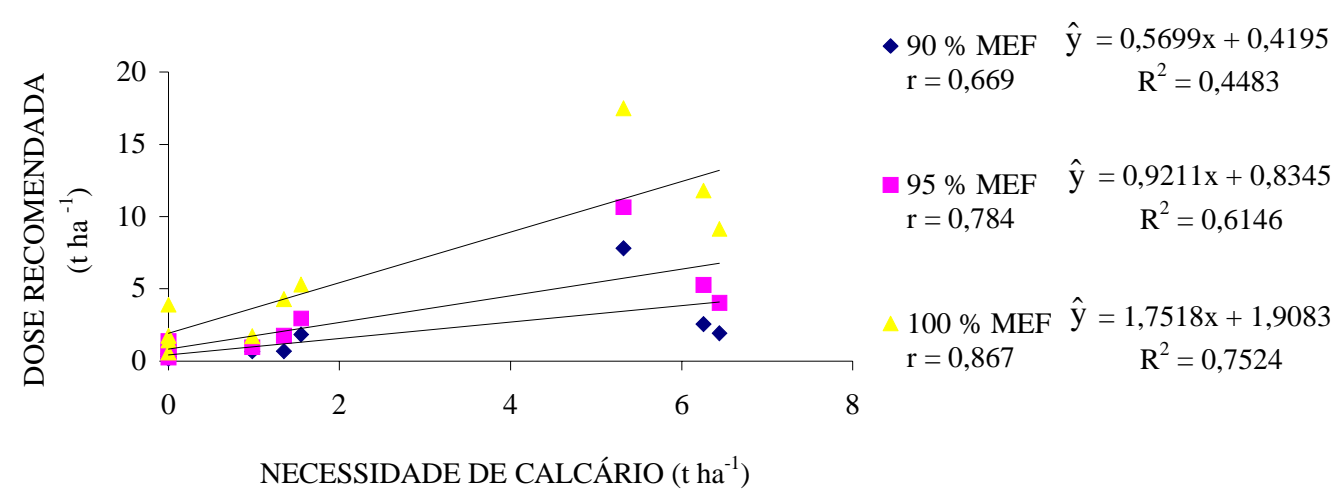

Figura 4. Relação entre as necessidade de calcário, pelo método baseado no teor de alumínio trocável mais cálcio e magnésio, e as doses recomendadas de calcário para obtenção de 90, 95 e 100\% da máxima eficiência física (ME F).

Método Baseado na Acidez Potencial $(\mathrm{H}+\mathrm{Al})$

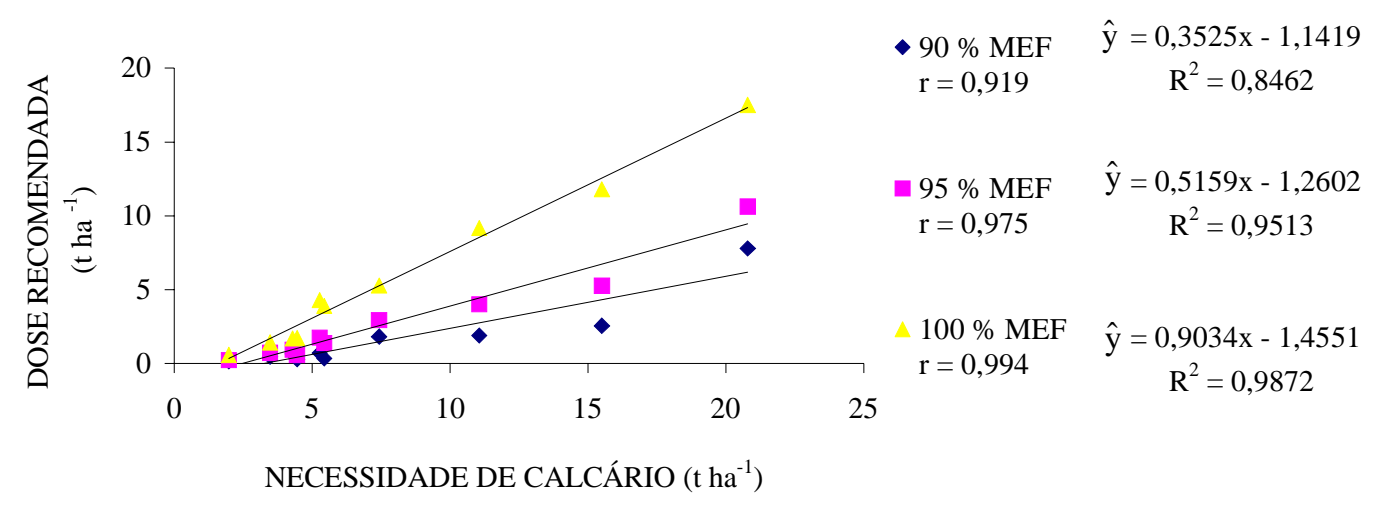

Figura 5. Relação entre as necessidades de calcário, pelo método baseado na acidez potencial $(\mathrm{H}+\mathrm{Al})$, e as doses recomendadas de calcário para obtenção de 90, 95 e 100\% da máxima eficiência física (MEF). 
Método Baseado na Saturação por Bases (70 \%)

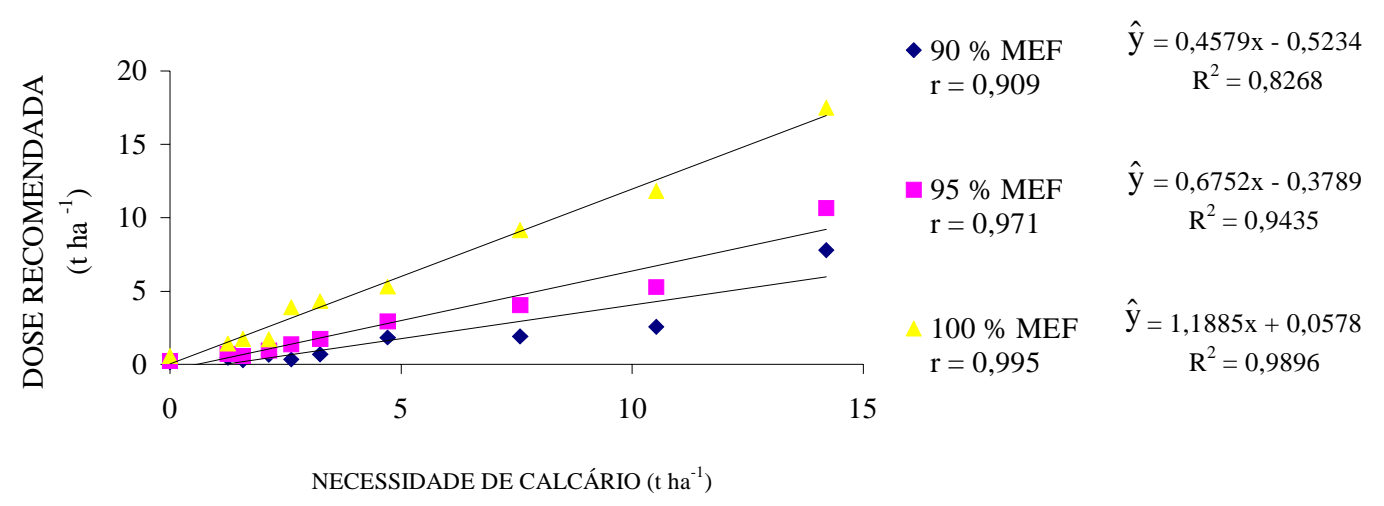

Figura 6. Relação entre as necessidades de calcário, pelo método que considera a saturação por bases para elevar V a 70\%, e as doses recomendadas de calcário para obtenção de 100, 95 e 90\% da máxiam eficiência física (MEF).

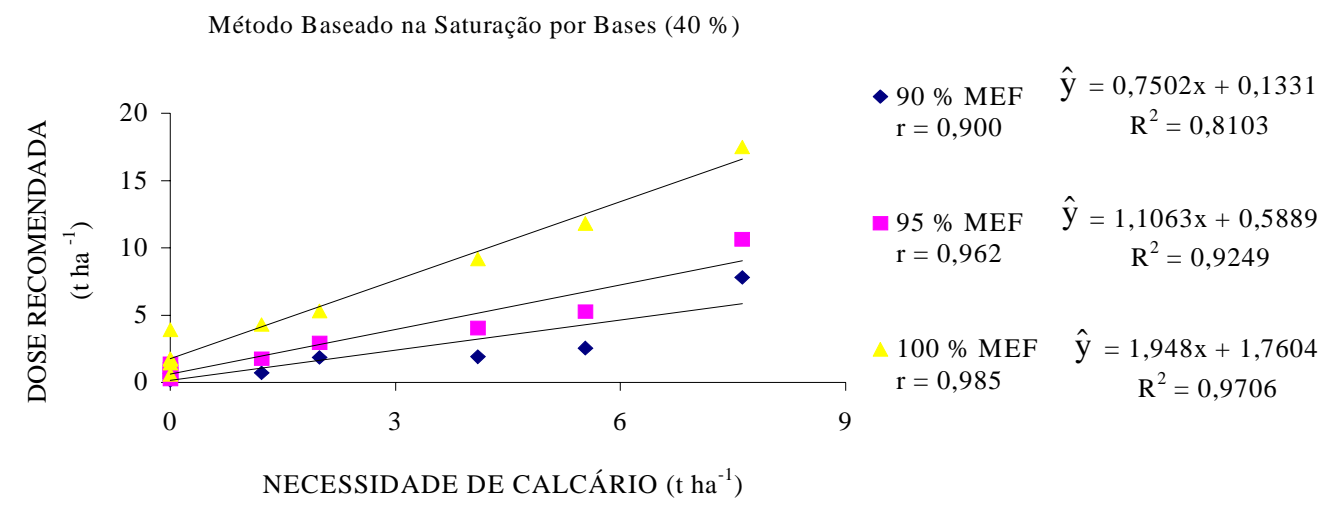

Figura 7. Relação entre as necessidades de calcário, pelo método que considera a saturação por bases para elevar V a 40\%, e as doses recomendadas de calcário para obtenção de 100, 95 e 90\% da máxima eficiência física (MEF).

\section{CONCLUSÕES}

1. As características dos solos que mais influenciaram recomendação da calagem, para o arroz em solos inundados, variaram com o critério adotado, se 90,95 ou $100 \%$ da MEF, para definir as doses recomendáveis. O carbono orgânico e a saturação por bases influenciaram o critério de $90 \%$ da MEF; de $95 \%$ foram o carbono orgânico e o teor de cálcio mais magnésio. J á a acidez potencial e o teor de cál cio mais magnésio influenciaram o critério de $100 \%$ da MEF.

2. Os teores de Fe e Mn redutíveis não tiveram efeito significativo na defini ção das doses de cal cário recomendáveis, indicando que o aumento da matéria seca, em função da calagem, não pode ser atribuído só ao controle da toxicidade de Fe, mas também ao fornecimento de $\mathrm{Ca}$ e $\mathrm{Mg}$ para as plantas e neutralização do Al3+ tóxico.

3. O método que visa elevar $\mathrm{V}$ a $40 \%$, conforme indicado pela Comissão de Fertilidade de Solo do Estado de Minas Gerais, proporcionou melhores estimativas das doses recomendadas para a obtenção de $95 \%$ da MEF. Contudo, em se considerando a calagem como uma prática de retorno econômico a médio e longo prazo, talvez os métodos baseados na acidez potencial e na saturação por bases (para elevar $\mathrm{V}$ a $70 \%$ ), que se aproximam mais das doses recomendadas pelo critério de $100 \%$ da MEF, sejam mais adequados. Tais considerações deverão ser confirmadas com trabal hos de cal i bração em campo, considerando a produção econômi ca de grãos, a longo prazo. 


\section{TERATURA CITADA}

BARBOSA FILHO, M.P.; FAGERIA, N.K. \& STONE, L.F. Manejo d'água e calagem em relação à produtividade e toxidade de ferro em arroz. Pesq. Agropec. Bras., 18:903-910, 1983.

BARBOSA FILHO, M.P. Toxicidade de ferro em arroz irrigado. In: REUNIÃO SOBRE FERRO EM SOLOS INUNDADOS, 1., Goiânia, 1987. Anais. Goiânia, EMBRAPA, 1988. p.205.

COMISSÃO DE FERTILIDADE DO SOLO DO ESTADO DE MINAS GERAIS. Recomendação para o uso de corretivo e fertilizantes em Minas Gerais. 3a aproximação. Belo Horizonte, EPAMIG, 1978. 80p.

DEFELIPO, B.V.; BRAGA, J.M. \& SPIES, C. Comparação entre métodos de determinação da necessidade de calcário de solos de Minas Gerais. Experientiae, 13:111-136, 1972. p.5.

EMPRESA BRASILEIRA DE PESQUISA AGROPECUÁRIAEMBRAPA. Serviço Nacional de Levantamento e Conservação do Solo. Manual de Métodos de Análise de Solo. Rio de J aneiro, 1979. não paginado.

FAGERIA, N.K.; BARBOSA FILHO, M.P. \& CARVALHO, J.R.P. de. Influência de ferro no crescimento e na absorção de $P$, $\mathrm{K}, \mathrm{Ca}$ e Mg pela planta de arroz em solução nutritiva. Pesq. Agropec. Bras., 16:483-488, 1981.

FAGERIA, N.K.; BARBOSA FILHO, M.P.; CARVALHO, J.R.P.; RANGEL, P.H.N.\& CUTRIM, V.A. Avaliação preliminar de cultivares de arroz para a tolerância à toxidez de ferro. Pesq. Agropec. Bras., 19:1271-1278, 1984.

FERNANDES, B. Retenção e movimento de água no solo. Viçosa: Universidade Federal de Viçosa, 1967. 48p. (Tese de Mestrado)

FREIRE, F.M.; NOVAIS, R.F.; SOARES, P.C.; COSTA, L.M. \& FARIA, E.A. Calagem, adubação orgânica e manejo da água no controle da toxicidade de ferro em arroz irrigado. R. Ceres, 32:162-169, 1985.

HOWELER, R. H. Iron-induced oranging disease of rice in relation to physico-chemical changes in a flooded oxisol. Soil Sci. Soc. Am. Proc., 37:898-903, 1973.

KAMISNKI, J .; XAVIER, F.M.; BARTZ, H.R. \& RHEINHEIMER, D.S. Efeito de calagem e da adubação potássica em cobertura sobre o rendimento de grãos de arroz, variedade BR-IRGA 409, irrigado por inundação. In: REUNIÃO BRASILEIRA DE FERTILIDADE DO SOLO E NUTRIÇÃO DE PLANTAS, 21., Santa Maria, 1990. Anais. Santa Maria, SBCS, 1990. p.96.

KEENEY, D.R. \& COREY, R.B. Factors affecting the lime requirements of Wisconsin soils. Soil Sci. Soc. Am. Proc., 27:277-280, 1963.

LEITE, N.; GARGANTINE, H.; HUNGRIA, L.S. \& IGUE, T. Efeitos de nitrogênio, fósforo, calcário e micronutientes em cultura de arroz irrigado no Vale do Paraíba. Bragantia, 29:273-285, 1970.

LINDSAY, W.C. \& NORVEL, W.A. Development of a DTPA soil test for zinc, iron, manganese and copper. Soil Sci. Soc. Am. J., 42:421-428, 1978.
MACHADO, M.O.; GOMES, A.S. \& PAULETTO, E.A. Resposta do arroz irrigado em quatro safras sucessivas à aplicação de fósforo e calcário dolomítico. In: REUNIÃO DA CULTURA DO ARROZ IRRIGADO, 13., Camburiú, 1984. Anais. Florianópolis, EMPASC, 1984. p.195-210.

MACHADO, M.O. Caracterização e adubação do solo. In: EMPRESA BRASILEIRA DE PESQUISA AGROPECUÁRIA. Fundamentos da cultura do arroz irrigado. Campinas, Fundação Cargil, 1985. p.129-179.

MALAVOLTA, E. Manual de química agrícola. São Paulo, Agronômica Ceres, 1959. 487p.

MELLO, J.W.V. Dinâmica de fósforo, ferro e manganês e disponibilidade de fósforo para o arroz em solos inundados. Viçosa, Universidade Federal de Viçosa, 1991. 212p. (Tese de Doutorado)

NYE, P.; CRAIY, D.; COLEMAN, N.T. \& RAGLAND, J.L. Ion exchange equilibrium involving aluminiun. Soil Sci. Am. Proc., 25:14-17, 1961.

OTTOW, J . C. G.; BENCKISER, G. \& WATANABE, G. I ron toxicity as a multiple nutritional soil stress. Trop. Agric. Res. Ser., 5:167-179, 1982.

RAIJ , B. van; CAMARGO, A.P.; CANTARELLA, H. \& SILVA, N.M. Alumínio trocável e saturação de bases como critérios para recomendação de calagem. In: CONGRESSO BRASI LEIRO DE CIÊNCIA DO SOLO, 17., Salvador, 1981. Resumos. Salvador, SBCS, 1981. p.49.

RAMOS, M.G.; ZANINI NETO, J.A.; MOREL, D.A.; NOLDIN, J.A.; MARQUES, L.F.; MIURAL, L.; SCHIMITT, A.T.; FROSI , J.F. \& MARQUES, L.F. Manual de produção arroz de irrigado. Florianópolis, EMPASC/EMATER/ACARESC, 1985. 225p.

SCHMIDT, N.C. \& GARGANTINI, H. Efeito da aplicação de calcário, matéria orgânica e adubos minerais em cultura de arroz, em solos de várzea irrigada. Bragantia, 29:293299, 1970.

SHOEMAKER, H.E.; MCLEAN, E.O. \& PRATT, P.F. Buffer methods for determining lime requirements of soil with apreciable amounts of extractable aluminum. Soil Sci. Soc. Am. Proc., 25:274-276, 1961.

SOUZA, D.M.G.; MIRANDA, L.N.; LOBATO, E. \& KLIEMAN, H.J . Avaliação de métodos para determinar as necessidades de calcário em solos de cerrado de Goiás e do Distrito Federal. R. Bras. Ci. Solo, 4:144-148, 1980.

TANAKA, A.; LOE, R. \& NAVASERO, S.A. Some mechanims involved in the development of iron toxity symptoms in the rice plant. Soil Sci. Plant Nutr., 12:32-38, 1966.

VAHL, L.C. Toxidez de ferro em genótipos de arroz irrigado por alagamento. Porto Alegre, Universidade Federal do Rio Grande do Sul, 1991. 173p. (Tese de Doutorado)

VETTORI, L. Métodos de análise de solo. Rio de J aneiro: E.P.E., 1969. 29p. (Boletim Técnico) 